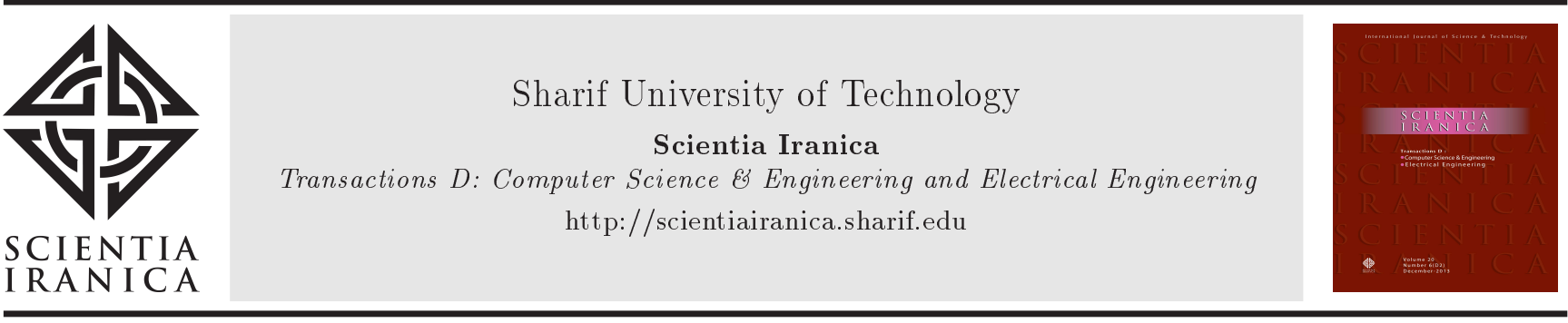

\title{
Electricity market assessment in wind energy integrated power systems with the potential of flexibility: A boundary condition approach
}

\author{
H. Gharibpour and F. Aminifar* \\ School of Electrical and Computer Engineering, College of Engineering, University of Tehran, Tehran, Iran.
}

Received 28 October 2019; received in revised form 9 December 2019; accepted 23 December 2019

KEYWORDS
Flexibility;
Equilibrium;
Wind energy;
Demand response
program;
Ramping capability.

\section{Introduction}

\subsection{Motivation}

There is no doubt that the world has been experiencing the ever-increasing installation of renewable energies. The tremendous transition to fill some parts of the world energy portfolio with green and environmentallyfriendly energy has added new complexity to the power systems. Generally, due to the intensive variation of the output power of variable renewable energy resources, the power systems fall short in terms of flexibility. Explicitly speaking, power systems suffer

\footnotetext{
*. Corresponding author.

E-mail address: faminifar@ut.ac.ir (F. Aminifar)
}

\begin{abstract}
This paper focuses on dynamic equilibrium considering the flexible ramp havior of different players is modeled throug a multi-leader-common-follower game, in which suppliers and demand response aggregators are laid as the leaders with the market o find equilibria in the real-time market is proposed. The effect of considering demand esponse resources and flexible ramp penalty price on the strategic behavior of players in the way for the formation of uplift payments.
\end{abstract}

(C) 2022 Sharif University of Technology. All rights reserved. from the lack of ramping-capable units in the future grids with a significant number of renewables. In most electricity markets, the vigorous variability of the output power of the resources has not been considered in the market design. Upon mitigating this issue, an efficient market must discriminate between flexible units against tenacious ones; otherwise, in order to handle the variation of the output power, major load shedding or wind spillage is inevitable.

System variation can be reflected in the net-load, which is load minus variable renewable energy sources. In many cases, the net-load has deeper valley, higher peak, and intensive ramp-up and ramp-down. Among the main resources that are able to manage variation and provide ramping flexibility are fast response units, demand response resources, and storage systems. In order to alleviate the ramping deficiency and exploit 
the ramping capability of flexible resources, there are mainly two different approaches taken by ISOs. Some of these solutions namely reforecasting of uncertain parameters close to the real-time operation and grid code enforcement are of out-of-market nature. To take advantage of the economic theorem, another way of flexibility provision comes across with electricity markets. What is so recognizable is that every service should be remunerated in such a way that its provision is uninterruptedly guaranteed within the system. Currently, in order to emit proper economic signals to invest in high ramping-capable units, or so-called agile units, California ISO and Mid-continent ISO have introduced a new product in their subdued markets $[1,2]$. This product is called Flexible Ramp Product (FRP) by the former, while in the latter, it is known as Ramp Capability Product (RCP).

Flexible ramp product has been introduced as a market-based solution to emit transparent price signal yielding investment on ramping capable resources and to facilitate the integration of variable renewable resources. Flexible ramp can be procured within the system by other services such as regulation product. It is worth mentioning that the regulation product cannot render proper price signal on agility because the main function of regulation service is to instantaneously balance the generating with load not to procure the ramp. An important point is that the inter-temporal nature of the flexible ramp product necessitates using a dynamic game approach. The proposed dynamic game is an effective model to simulate the strategic behavior of players in a well-designed market.

This paper is categorized as follows. The contribution of this paper is clarified in the next part. The strategic behavior of players in the electricity market concerning FRP is surveyed in Subsection 1.3. In the remaining part of Section 1, demand response programs are then focused. Solution approach is explained in Section 2. Complete problem formulation is brought in Section 3. Finally, simulation studies and conclusions are made in Sections 4 and 5, respectively.

\subsection{Contribution}

In this paper, the effect of flexible ramp market on the player's strategic behavior in the real-time market is studied. Due to the inter-temporal nature of flexible ramp constraints, a dynamic equilibrium based on receding horizon is proposed. Demand response programs are also included into the model. The main contributions of this paper are as follows:

- Simultaneously accommodating flexible ramp market and demand response resources to alleviate ramping deficiency in the context of Mathematical Program with Equilibrium Constraints (MPEC) and Equilibrium Problems with Equilibrium Constraints (EPEC);
- Evaluating the effect of FRP violation and characterizing the inter-temporal nature of the problem into decision-making process;

- Proposing a dynamic forward rolling process to find equilibria in the real-time market.

\subsection{Players' Strategic Behavior and FRP}

In the restructured electric power system, the strategic behavior of the players is studied based on the equilibrium concept. In the game theory context, exploring strategic behavior of players can be modeled as a game to seek the Nash Equilibrium (NE), a level at which no player is unilaterally interested to change its strategy to secure higher pay-off.

The strategic behavior of the electricity market players has been studied in many studies. Electricity market environment in which buyers can maximize profits by making coalition is modeled as a game in [3]. The paper evaluates the bidding strategy of market participants on the buyer side and does not model the strategic behavior of sellers.

There are several studies in the literature that focus on finding equilibrium through the Supply Function Equilibrium (SFE) [4-6]. Reference [4] is one of the most pioneer articles that considered wind uncertainty. In [5], the asymmetric supply function was presented to model the load uncertainty with continuous probability distribution function. SFE was numerically estimated through an approach with piecewise linear supply functions.

In [7], virtual power plant including wind units and energy storage facilities were considered as a price taker which could buy and sell energy in both dayahead and real-time markets. To manage the uncertainties of wind and market prices, two-stage robust optimization was exploited. The bidding strategy for day-ahead and real-time market was prescribed at the first and second stages, respectively. Virtual power plant strategic role as a price maker did not receive focus in [7]. The purpose of authors in [8] was to determine the optimal bidding strategy for the riskaverse demand-side resources as a price taker in the day-ahead market. This study presented a model for aggregating demand-side sources. Therefore, there was no need for modeling each type of load response sources in an individual fashion. The real-time market was not incorporated in the modeling. To find NE strategies in a two-settlement electricity market, a competitive coevolutionary algorithm was proposed in [9], where the strategic interactions of market agents were modeled as a non-cooperative game. A bi-level bidding strategy optimization model in a transactive energy market was proposed in [10] in which the clearing of the transactive energy market was considered as the lower level problem. This study [11] proposed an intraday risky power market enabling the wind power producers 
to mitigate their real-time power mismatches. To do so, a three-settlement electricity market was studied using EPEC and the equilibrium was analyzed.

In [12], several agents could affect the price signal. The model chosen for each agent was MPEC. The behavior of all agents was reflected in EPEC. The effect of variable and stochastic resources on the equilibrium was not investigated in the paper. Also, the model is single-time and not capable to accommodate the ramping constraints. Unlike iterative methods, EPEC is a set of constraints that can explicitly model the equilibrium. This model is based on the strong duality theory and KKT conditions. In fact, in the literature, EPEC is considered as a multi-leader-multifollower game where the market participants act as the leader because they start the game by submitting their offers/bids. Then, the market operator follows the game by starting the process of clearing the market; hence, it plays the game as the common follower.

The role of flexible ramp product, which is procured at each interval, is to guarantee the procurement of needed ramps at the following interval. Recently, the effect of flexible ramp product on the market operations has been investigated in several papers. Cornelius [13] used a modified unit commitment and economic dispatch to explore the economic and reliability aspects of RCP introduced in the MISO electricity market. Baseline model and a RCP-included model as two practical programs, which are currently used by MISO, were formed. The effect of RCP on the scheduling program was studied. It was concluded that ramp-up product did its best in alleviating system ramp shortage. In order to better predict the maximum available flexibility within the power system, the constraints with less than one-hour characteristics were included in an hourly-based security-constraint unit commitment [14]. In [2], a mathematical formulation of FRP was presented that was actually used in the CAISO market. The above study took into consideration the flexible ramp constraint in the re-dispatch process. In order to meet the system ramp requirement in the presence of wind power, flexible ramp constraints in the dayahead and reserve markets were modeled in [15]. In the research, an adjustable interval optimization was utilized to handle the uncertain parameters. The proposed model could reduce the conservative level by setting the upper and lower limits of wind power prediction. In order to investigate the market price in the presence of high renewable energy, a multiinterval Cournot equilibrium respecting flexible ramp market was investigated in [16]. Only the hydro and thermal units can strategically participate in the energy and flexible ramp markets, which are simultaneously settled; of note, all players are referred to as the market price takers and they can only decide on the amount of their supply. Variable renewable energies have not received much attention as the sources of flexible ramp suppliers, while wind turbines can be controlled and are able to change their power output well [17]. In [18], the role of wind power plants was studied as the supplier of FRP. This study ultimately concludes that both the system and wind resources can benefit from this cooperation.

Considering a wind power ramping product, a multi-timescale unit commitment and economic dispatch model was presented in [19]. To alleviate the system ramping deficiency, the capability of wind power in ramp procurement was utilized. The aforementioned study employed an optimized swinging door algorithm to determine and forecast wind power ramps. Finally, it was concluded that wind power ramping product would decrease the production cost and improve the reliability of power system operations. To deal with the stochastic nature of market operation and especially intensive variation of net-load in a system with a highpenetrated level of variable resources, exploitation of flexible resources as well as the incorporation of a sub-hourly flexible ramp market were emphasized in [20]. To obtain the optimal schedule of the energy, reserve, and flexible ramp product, a stochastic dayahead market-clearing model was developed. Having taken energy and FRP into consideration, this study [21] investigated the strategic behavior of wind power producers. A static game was employed to model the player's interaction. The effect of considering FRP on the existence of electricity market equilibrium was explored in [22], where the strategic behaviors of players were modeled in the form of a stochastic equilibrium. In order to appraise the effect of FRP on the market, a Nash-Cournot approach was exploited in [23]. This research modeled FRP in the day-ahead stage. The authors eventually came to the conclusion that FRP could result in facilitating the integration of renewable energies into power system.

\subsection{Demand response programs}

According to EIA (US Energy Information Administration) definition, demand-side management programs consist of the planning, implementation, and monitoring activities of electric utilities that have been designed to encourage consumers to modify their level and pattern of electricity usage [24]. Conventionally, the significance of the demand response programs is summarized as decreasing load shedding amounts, postponing investment cost of new power plant construction, stabilizing market prices, and reducing the total electricity cost $[25,26]$. Demand Response Programs (DRPs) can not only play important roles in the mentioned items but also facilitate the integration of variable renewable energy resources into power systems. Demand response resources can provide ancillary services to absorb net-load disruptions and replace 
the expensive fast-ramping reserve generating units [27]. In many papers, DRPs are mainly categorized into two main groups [28,29]: price-based programs and incentive-based programs. In the former, demands adjust their load level based on the electricity price signals and include time-of-use, real-time, and critical peak pricing programs. The latter, itself, is divided into voluntary, mandatory, and market-based programs. Voluntary programs include emergency demand response and direct load control programs. Interruptible/curtailable services and capacity market programs are among the mandatory ones in which enrolled demands are penalized if they do not act based on their commitments. Finally, demand bidding and ancillary service programs fall within market-based ones. Demand bidding programs allow demand-side players to participate in wholesale electricity market and adjust their bids based on the amount of elasticity. It is also a common practice to take advantages of demand-side capabilities in providing ancillary services such as operating reserve. Simultaneous provision of flexible ramping product by interruptible loads was investigated in [30]. In the paper, incentive compatible contracts were designed to encourage customers to reveal their true outage costs.

In [31], the role of thermal power plants in a power system was presented with high penetration of variable generating. Herein, different sources of demand-side flexibility were considered. A generating planning model combined with a unit commitment and economic dispatch model were employed for conducting this research. In [32], an optimization model was proposed for a battery energy storage aggregator to procure FRP in day-ahead energy and reserve markets. Then, adoption of strategies for battery energy storage aggregators to participate in the electricity markets was emphasized.

Considering fast-ramping units, demand response, and energy storage, the referenced study [33] aimed to provide a systematic approach for evaluating the level of flexibility of a power system. It considered an 'online' index to evaluate the technical aptitude of the fast-ramping units, demand response, and energy storage to deliver the required flexibility.

To deal with the uncertainty and intermittency of wind power, demand-side participation was considered in [34]. An optimization model offered for aggregated wind power and flexible loads in day-ahead electricity markets was suggested. It was concluded that flexible load could cover wind power imbalance. A practical analysis in Germany regarding the use of demand response as reserve capacity was conducted in [35]. It was demonstrated that using the potential of different types of demand response resources together with enhanced wind power predictions could bound the additional imbalance costs in Germany.

\section{Solution approach}

In this paper, fast-response units and demand response resources are utilized to meet the system ramp requirement. Demand can replicate elasticity with demand bidding program in the energy market. In addition, ancillary service programs are employed to alleviate ramping deficiency. To this end, the marketbased DRPs are emphasized. The proposed formulation is written such that firms' strategic behavior can be investigated at once, while each firm can own a variety of energy resources and even demand response ones. Figure 1 shows the entities and their roles in the proposed formulation. Demand Response Aggregators (DRAs) are responsible to participate in demand bidding program (wholesale electricity market) on behalf of demand response resources. In fact, DRAs can identify and contract with small-sized demand response resources and participate on their behalf as a larger party in the wholesale electricity market. Their capability to provide a flexible ramp is also modeled considering their role as a flexible ramp provider. By participating in flexible ramp market, DRAs as commercial firms can make profit while alleviating power system operator's concern regarding the ramping deficiency. Among the generating units, both fast response units, here Ramping-Capable Units (RCUs), and conventional units supply energy; however, only RCUs can make profit with flexible ramp procurement. In addition, energy storage systems enjoy a great capability to alleviate ramping deficiency, but their potential flexibilities are left to be fully digested in future works.

\subsection{Boundary condition equilibrium}

In this study, the real-time energy and flexible ramp markets are simultaneously cleared every 15 minutes where a sequence of four 15-minute intervals is considered in every run of real-time market. The approach taken here works on a receding horizon basis and can be well-matched with advanced market practices such as CAISO, which is using rolling horizon optimization. CAISO runs real-time market at a 5-minute interval and looks ahead for at least a 120-minute time horizon (11 intervals ahead) [36]. At the beginning of the rolling

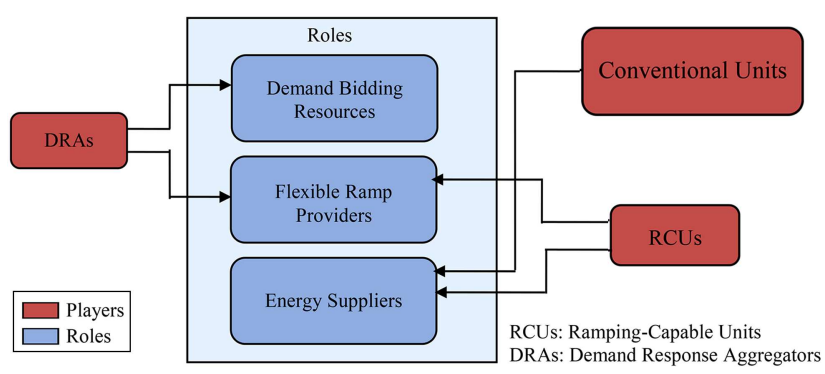

Figure 1. Entities and their roles. 


$$
- \text { Minimizingg } \Xi_{\Xi L}\left\{\begin{array}{l}
\sum_{t, i \in \Omega_{j}}\left(\lambda_{t}-\lambda_{i}^{G} \times \Delta\right) \times P_{i, t}^{G}-\sum_{t, d \in \Omega_{j}}\left(\lambda_{d}^{D} \times \Delta-\lambda_{t}\right) \times P_{d, t}^{D}+ \\
\sum_{t<T, i \in \Omega_{j}} \mu_{t}^{U} \times U R_{i, t}^{G}+\sum_{t<T, i \in \Omega_{j}} \mu_{t}^{D} \times D R_{i, t}^{G}+ \\
\sum_{t<T, d \in \Omega_{j}} \mu_{t}^{U} \times U R_{d, t}^{D}+\sum_{t<T, d \in \Omega_{j}} \mu_{t}^{D} \times D R_{d, t}^{D}
\end{array}\right\}
$$

Box I

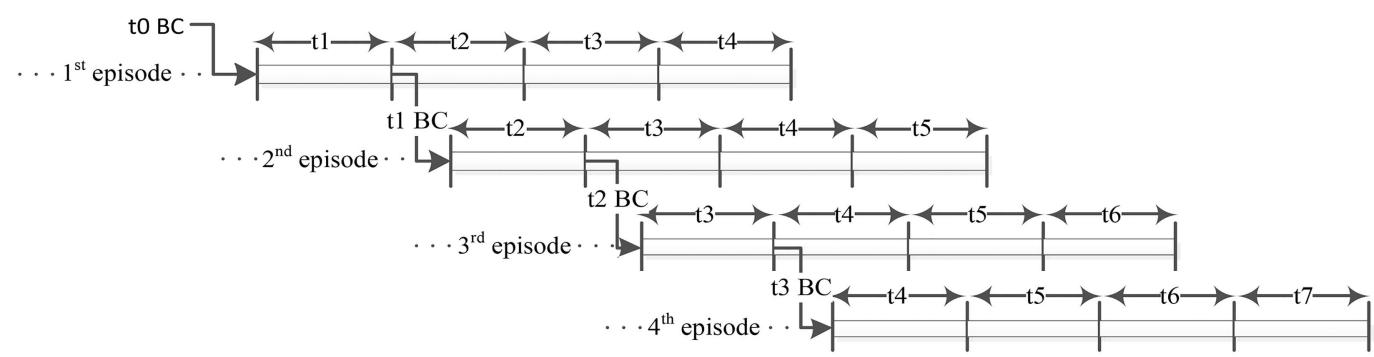

Figure 2. Dynamic Boundary Condition (BC) approach.

process (hereafter, each of this rolling proses is called an episode), the initial condition of output power of generating units, consumed power of DRAs, Flexible Ramp-Down (FRD), and Flexible Ramp-Up (FRU) supplied by generating units, FRP, and FRD supplied by DRAs are considered as the problem boundary conditions for the first-time interval. Then, bi-level optimization problems, one per each firm, are proposed to model the interaction between market and agents. At the first level of each of these bi-level problems, the profit of the players is maximized according to decision variables (real-time and flexible ramp product prices) derived from the second level, which replicates the market clearing process in the real-time market. Based on the strong duality theory, the second level of the bi-level problems is replaced by its primal-dual equations. In doing so, a bi-level optimization problem is cast into a single level. This single level optimization problem is then recast into a suit of constraints by applying KKT condition. Joint consideration of these suits of constraints, for each firm, results in EPEC. In other words, EPEC includes the best responses of all players that are the strategies that produce the most favorable outcomes from all players and hence, players are no longer interested in changing their strategy. In fact, the constituent elements of EPEC are as follows [22]:

- MPEC primal equalities;

- MPEC primal inequalities;

- MPEC dual equalities (the set of derivatives of Lagrange function of MPECs to all primal variables);
- MPEC dual inequalities;

- The set of complementary slackness of MPECs all inequalities.

The equilibrium points of the first-time interval in the first episode are then passed to the second episode as the boundary conditions for the second-time interval. EPEC model is now solved for the second episode. Consequently, the equilibrium points of the second episode are ready to initialize the third episode as the boundary conditions and so on. The full process, which is shown in Figure 2, is continued for the fourth episode, too. Finally, equilibria are characterized by all these boundary conditions.

\section{Complete problem formulation}

The bi-level model for the $j$ th producer is defined as follows:

Eq. (1) is shown in Box I.

$$
\begin{aligned}
& 0 \leq \alpha_{i, t}^{G} \quad \forall i, t \\
& 0 \leq \alpha_{d, t}^{D} \quad \forall d, t \\
& \left\{\lambda_{t}, P_{i, t}^{G}, P_{d, t}^{D}, \mu_{t}^{U}, \mu_{t}^{D}, U R_{i, t}^{G}, D R_{i, t}^{G}, U R_{d, t}^{D}, D R_{d, t}^{D}\right\} \\
& =\operatorname{Arg}\{\operatorname{minimizing} \Delta \\
& \quad \times \sum_{t}\left(\begin{array}{l}
\sum_{i} \alpha_{i, t}^{G} \times P_{i, t}^{G}-\sum_{d} \alpha_{d, t}^{D} \times P_{d, t}^{D}+ \\
F P \times\left(S_{t}^{U}+S_{t}^{D}\right)
\end{array}\right)
\end{aligned}
$$




$$
\begin{aligned}
& \sum_{d} P_{d, t}^{D}-\sum_{i} P_{i, t}^{G}+N L_{t}^{E X}=0: \lambda_{t} \forall t, \\
& P_{i, t+1}^{G}+U R_{i, t}^{G} \leq \overline{P_{i}^{G}}: \gamma_{i, t}^{\mathrm{Gmax}} \forall i, t<T, \\
& \underline{P_{i}^{G}} \leq P_{i, t+1}^{G}-D R_{i, t}^{G}: \gamma_{i, t}^{\mathrm{Gmin}} \forall i, t<T, \\
& P_{i, t+1}^{G}-P_{i, t}^{G}+U R_{i, t}^{G} \leq R R_{i}^{G} \quad: \delta_{i, t}^{\mathrm{G}, \mathrm{U}} \forall i, t<T, \\
& P_{i, t}^{G}-P_{i, t+1}^{G}+D R_{i, t}^{G} \leq R R_{i}^{G}: \delta_{i, t}^{\mathrm{G}, \mathrm{D}} \forall i, t<T, \\
& P_{i, t 1}^{G}+U R_{i, \mathrm{t} 0}^{G} \leq \overline{P_{i}^{G}}: \gamma_{i}^{\mathrm{Gmax}, \mathrm{ini}} \forall i, \\
& \underline{P_{i}^{G}} \leq P_{i, \mathrm{t1}}^{G}-D R_{i, t 0}^{G}: \gamma_{i}^{\mathrm{Gmin}, \mathrm{ini}} \forall i, \\
& P_{i, t 1}^{G}-P_{i, t 0}^{G}+U R_{i, t 0}^{G} \leq R R_{i}^{G}: \delta_{i}^{\mathrm{G}, \mathrm{U}, \mathrm{ini}} \forall i, \\
& P_{i, t 0}^{G}-P_{i, t 1}^{G}+D R_{i, t 0}^{G} \leq R R_{i}^{G}: \delta_{i}^{\mathrm{G}, \mathrm{D}, \mathrm{ini}} \forall i, \\
& 0 \leq U R_{i, t}^{G} \leq R R_{i}^{G}: \theta_{i, t}^{\mathrm{Gmin}, \mathrm{U}}, \theta_{i, t}^{\mathrm{G} \max , \mathrm{U}} \quad \forall i, t<T,
\end{aligned}
$$$$
0 \leq D R_{i, t}^{G} \leq R R_{i}^{G}: \theta_{i, t}^{\mathrm{Gmin}, \mathrm{D}}, \theta_{i, t}^{\mathrm{Gmax}, \mathrm{D}} \quad \forall i, t<T,
$$$$
P_{d, t+1}^{D}+D R_{d, t}^{D} \leq \overline{P_{d}^{D}}: \gamma_{d, t}^{\operatorname{Dmax}} \forall d, t<T
$$$$
\underline{P_{d}^{D}} \leq P_{d, t+1}^{d}-U R_{d, t}^{d}: \gamma_{d, t}^{\text {Dmin }} \forall d, t<T,
$$$$
P_{d, t}^{D}-P_{d, t+1}^{D}+U R_{d, t}^{D} \leq R R_{d}^{D}: \delta_{d, t}^{D, U} \quad \forall d, t<T,
$$$$
P_{d, t+1}^{D}-P_{d, t}^{D}+D R_{d, t}^{D} \leq R R_{d}^{D}: \delta_{d, t}^{D, D} \quad \forall d, t<T,
$$$$
P_{d, t 1}^{D}+D R_{d, t 0}^{D} \leq \overline{P_{d}^{D}}: \gamma_{d}^{\text {Dmax,ini }} \forall d,
$$$$
\underline{P_{d}^{D}} \leq P_{d, t 1}^{d}-U R_{d, t 0}^{d}: \gamma_{d}^{\text {Dmin,ini }} \forall d,
$$$$
P_{d, t 0}^{D}-P_{d, t 1}^{D}+U R_{d, t 0}^{D} \leq R R_{d}^{D}: \delta_{d}^{\mathrm{D}, \mathrm{U}, \mathrm{ini} \quad \forall d,}
$$$$
P_{d, t 1}^{D}-P_{d, t 0}^{D}+D R_{d, t 0}^{D} \leq R R_{d}^{D}: \delta_{d}^{\mathrm{D}, \mathrm{D}, \mathrm{ini}} \quad \forall d,
$$$$
0 \leq U R_{d, t}^{D} \leq R R_{d}^{D}: \theta_{d, t}^{\text {Dmin, } \mathrm{U}}, \theta_{d, t}^{\text {Dmax }, \mathrm{U}} \quad \forall d, t<T,
$$

$$
0 \leq D R_{d, t}^{D} \leq R R_{d}^{D}: \theta_{d, t}^{\mathrm{Dmin}, \mathrm{D}}, \theta_{d, t}^{\mathrm{Dmax}, \mathrm{D}} \quad \forall d, t<T,
$$

$$
\begin{aligned}
& F R U P_{t} \leq \sum_{i} U R_{i, t}^{G}+\sum_{d} U R_{d, t}^{D}+S_{t}^{U}: \mu_{t}^{U} \quad \forall t<T, \\
& F R D P_{t} \leq \sum_{i} D R_{i, t}^{G}+\sum_{d} D R_{d, t}^{D}+S_{t}^{D}: \mu_{t}^{D} \quad \forall t<T, \\
& \quad(27)
\end{aligned}
$$

$$
\left.0 \leq S_{t}^{D}, S_{t}^{U} \quad: \zeta_{t}^{D}, \zeta_{t}^{U} \quad \forall t<T\right\}
$$

The $j$ th firm profit maximization (here minus of minimization) is the objective function at the upper level where $E^{U L}$ includes the prices offered for the energy to be supplied or consumed. The first two terms in Relation (1) are the $j$ th firm profit secured by both the producers and DRAs participating in the real-time energy market. The next four terms show the incomes maintained in the FRU and FRD markets by both the generating units and demand utilities that the firm owes, respectively. The nonnegativity of the prices offered for both generating units and DRAs is expressed by Relations (2) and (3), respectively. The lower level problem that simulates the real-time market clearance is cast in Relations (4)(28), where Relation (4) replicates the minus social welfare plus the violation cost of system ramp requirements. Power balance is enforced by Relation (5), where parametric expected net-load should be met. Putting aside the capacity for the possible use of ramp-up and ramp-down capabilities at the following interval, Relations (6) and (7) bound the output power of generating units, respectively. The same argument holds in the case of maximum ramp-up and ramp-down of generating units. To this end, Relations (8) and (9) are responsible for limiting maximum ramp-up and ramp-down of generating units. Considering boundary conditions, Relations (10)-(13) stand for Relations (5) and (6), respectively. In the mentioned constraints, the boundary conditions are no longer decision variables as they were in the previous episode. The capacities of ramp-up and ramp-down capability of generating units are limited by their maximum ramp-rates in Relations (14) and (15), respectively. Having reserved the capacity for possible use of ramp-up and rampdown capabilities, Relations (16) and (17) restrict the consumed power of DRAs by their minimum and maximum capacities, respectively. Maximum rampup and ramp-down of the consumed power of DRAs are bounded in Relations (18) and (19), respectively. Analogous to generating units, Relations (20)-(23) are responsible to initialize the consumed power of DRAs and stand for their counterparts in Relations (16)-(19), respectively. The capacities of ramp-up and rampdown capability of demands are limited by their maximum ramp-rates in Relations (24) and (25), respectively. System requirements for ramp-up and rampdown are met in Relations (26) and (27), respectively, considering slack variables. Finally, the non-negativity of ramp-up and ramp-down slack variables is observed in Relation (28).

Now, it is time to apply boundary condition approach, which is explained in Section 2, on the proposed model. The flowchart of the complete solution approach is depicted in Figure 3. 


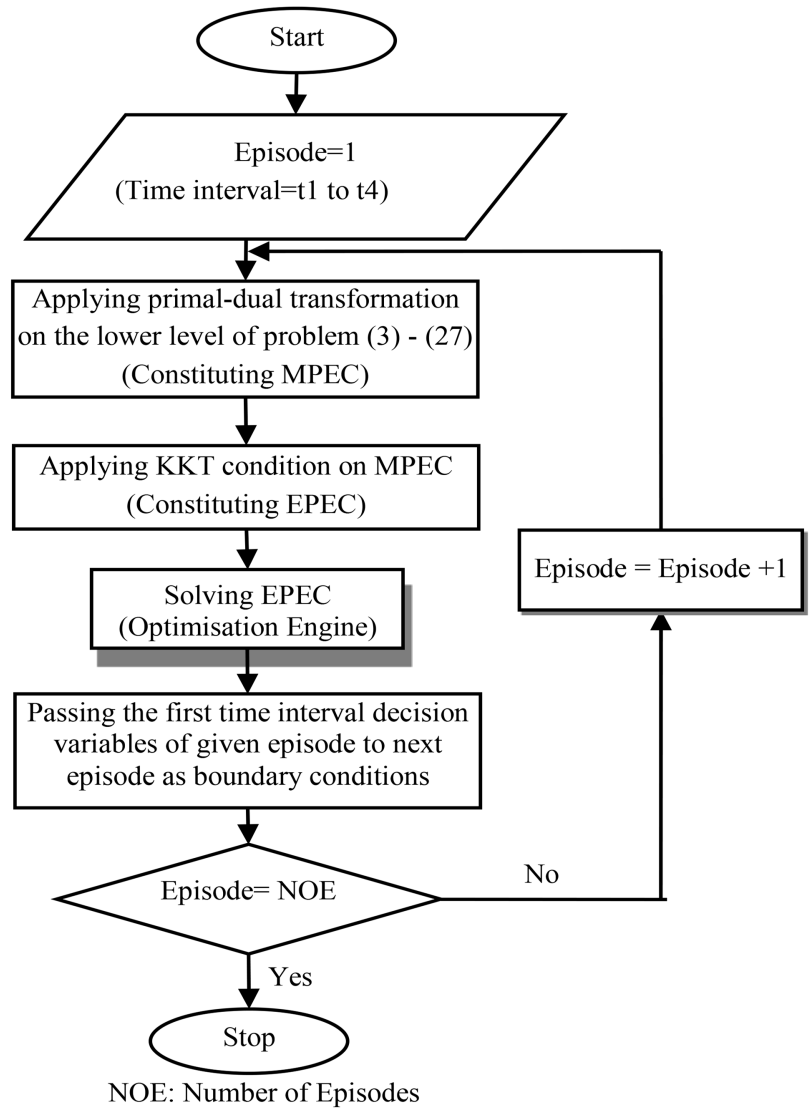

Figure 3. Dynamic Boundary Condition (BC).

\section{Simulation and results}

The effectiveness of the proposed methodology is verified through a three-firm system with 6 generating units and two demand response aggregators. The characteristics of generating units and DRAs are given
Table 2. The boundary conditions for the generating units.

\begin{tabular}{ccccccc}
\hline & \multicolumn{6}{c}{ Generating units } \\
\cline { 2 - 7 } & $\boldsymbol{i 1}$ & $\boldsymbol{i 2}$ & $\boldsymbol{i 3}$ & $\boldsymbol{i 4}$ & $\boldsymbol{i 5}$ & $\boldsymbol{i 6}$ \\
\hline$P_{i, t 0}^{G}$ & 200 & 100 & 200 & 100 & 100 & 200 \\
$U R_{i, t 0}^{G}$ & 0 & 0 & 0 & 5 & 15 & 15 \\
$D R_{i, t 0}^{G}$ & 0 & 0 & 0 & 5 & 15 & 15 \\
\hline
\end{tabular}

Table 3. The boundary conditions for demands.

\begin{tabular}{cccc}
\hline DRAs & $P_{d, t 0}^{D}$ & $U R_{d, t 0}^{D}$ & $D R_{d, t 0}^{D}$ \\
\hline $\boldsymbol{d} \mathbf{1}$ & 100 & 9 & 9 \\
$\boldsymbol{d} \mathbf{2}$ & 50 & 5 & 5 \\
\hline
\end{tabular}

in Table 1. It should be noted that a four-episode and seven fifty-minute time intervals, as shown in Figure 2, are considered. The boundary conditions in the first episode for generating units and demands are given in Tables 2 and 3, respectively. The system ramp-down and ramp-up requirements at each time interval are given in Table 4. Finally, the net-load is characterized in Figure 4. According to Figure 4, net-load includes two scenarios, namely ascending and descending scenarios. The scenario probabilities are 0.6 and 0.4 for ascending and descending scenarios, respectively.

Since the proposed methodology is a mixedinteger program, the simulation is done using a wellknown CPLEX solver under GAMS environment.

In order to evaluate the effectiveness of the proposed methodology, two different cases are modeled. In

Table 1. The characteristics of the generating units.

\begin{tabular}{|c|c|c|c|c|c|c|c|c|}
\hline \multirow[b]{2}{*}{ Characteristics } & \multicolumn{2}{|c|}{ DRAs } & \multicolumn{6}{|c|}{ Generating units } \\
\hline & $d 1$ & $d 2$ & $i 1$ & $i 2$ & $i 3$ & $i 4$ & $i 5$ & $i 6$ \\
\hline Ownership St & $j 3$ & $j 3$ & $j 1$ & $j 2$ & $j 1$ & $j 1$ & $j 2$ & $j 2$ \\
\hline$\underline{P_{i}^{G}}, \overline{P_{i}^{G}}$ & - & - & 100,300 & 50,150 & 50,250 & 50,150 & 10,110 & 50,350 \\
\hline$\underline{P_{d}^{D}}, \overline{P_{d}^{D}}$ & 0,200 & 0,400 & - & - & - & - & - & - \\
\hline$\lambda_{i}^{G}$ & - & - & 10 & 11 & 18 & 19 & 20 & 21 \\
\hline$\lambda_{d}^{D}$ & 25 & 30 & - & - & - & - & - & - \\
\hline$R R_{i}^{G}$ & - & - & 0 & 20 & 20 & 20 & 60 & 80 \\
\hline$R R_{d}^{D}$ & 75 & 50 & - & - & - & - & - & - \\
\hline
\end{tabular}




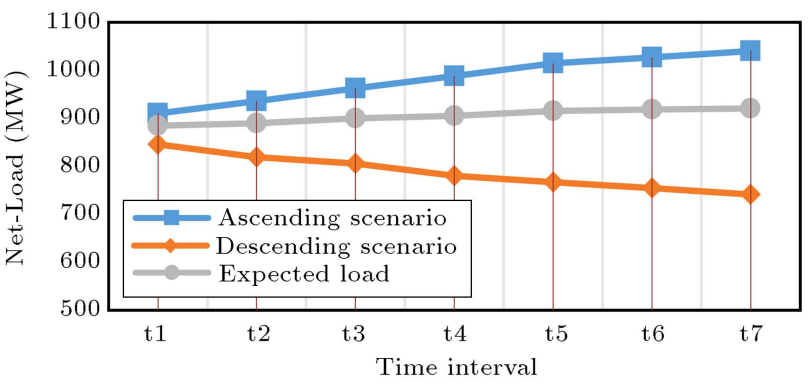

Figure 4. Net-load at different time intervals.

Table 4. System ramp-down and ramp-up requirements.

\begin{tabular}{ccccccc}
\hline & \multicolumn{6}{c}{ Time intervals } \\
\cline { 2 - 7 } & $\boldsymbol{t 1}$ & $\boldsymbol{t 2}$ & $\boldsymbol{t 3}$ & $\boldsymbol{t 4}$ & $\boldsymbol{t 5}$ & $\boldsymbol{t 6}$ \\
\hline$F R D P_{t}$ & 75.4 & 96.2 & 122.2 & 130 & 136.5 & 149.5 \\
$F R U P_{t}$ & 81.9 & 96.2 & 122.2 & 130 & 136.5 & 149.5 \\
\hline
\end{tabular}

Table 5. Firms' income in cases I and II.

\begin{tabular}{cccc}
\hline & \multicolumn{3}{c}{ Firms } \\
\cline { 2 - 4 } Case & $\boldsymbol{j} \mathbf{1}$ & $\boldsymbol{j 2}$ & $\boldsymbol{j 3}$ \\
\hline Case I & 3622 & 1266 & 267 \\
Case II & 3766 & 1816 & 602 \\
\hline
\end{tabular}

the first case, demand function for ramp-up and rampdown (or in another word, the penalty price for ramp violation (FP)) is considered $100 \$ / \mathrm{MWh}$, while in the second one, there is no price cap on ramp penalty. In doing so, the effect of considering the demand function for flexible ramp can be investigated accordingly.

Table 5 shows the firms' income in each case. Compared to the first case, firms' income increases in the second case in which there is no imposed demand
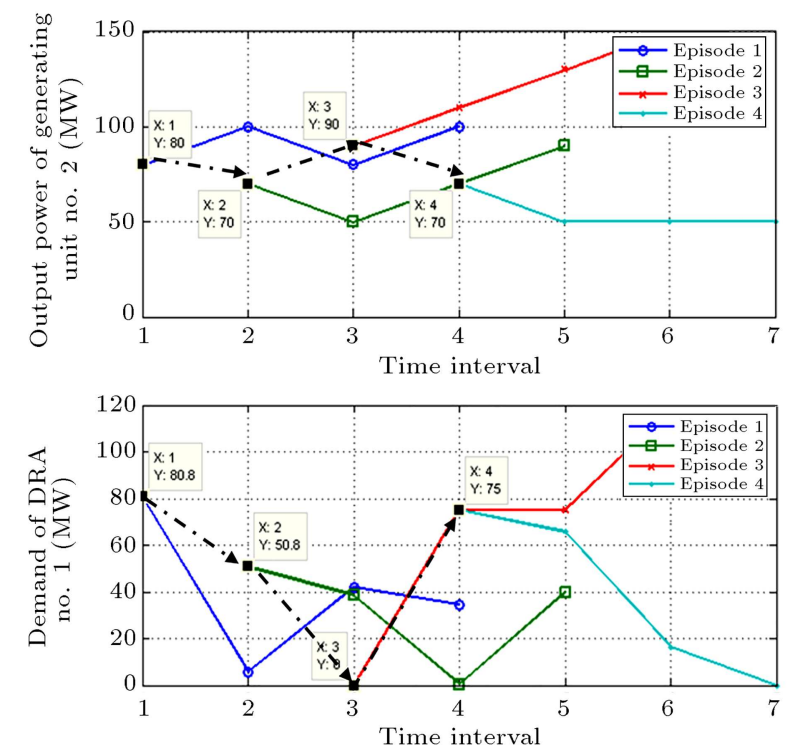

Figure 5. The output power of the generating units 2 and 6 and the demand level of DRAs. Dash-dotted arrows show the trend of the amount of power, which should be produced or consumed in real-time operation in equilibrium. function for the FRU and FRD products. This fact thoroughly is consistent with our expectation. Imposing any cap, whether on energy prices or ancillary services, can be interpreted as market manipulation. In order to avoid rocket-sky-price events, which result in social and political issues and can guarantee investment in all types of generating units, many electricity markets set a cap on their electricity market. It is proved that cap price causes all generating units to lose a fixed part of their incomes. To this end, setting a cap on energy market necessitates the establishment of the capacity payment mechanisms or uplift payments. Upon comparing the firms' income in two cases, the differences between two incomes can be construed as uplift payments that should be paid by stakeholders. Maintaining such payments in electricity markets can guarantee the sustainability of energy procurement along with the ramping capability. Otherwise, the ramp procurement in the system is faced with shortage after a while.

The results associated with case I are given in Figures 5 to 7 . The output power of the generating units 2 and 6 and the demand level of DRAs are shown in Figure 5. In each episode, the data tip shows the boundary condition for the next episode. As mentioned in the solution approach section, these boundary conditions are known as the equilibria. The boundary conditions in each episode are also responsible to initialize the associated next episode. The interesting point is that in some cases, the equilibrium points exactly coincide with each episode trend. For example, considering the demand level of the first DRA, the boundary conditions in episodes 3 and 4 are exactly stretched to the trend of episode 3 . Regarding the demand level of the second DRA, the same thing
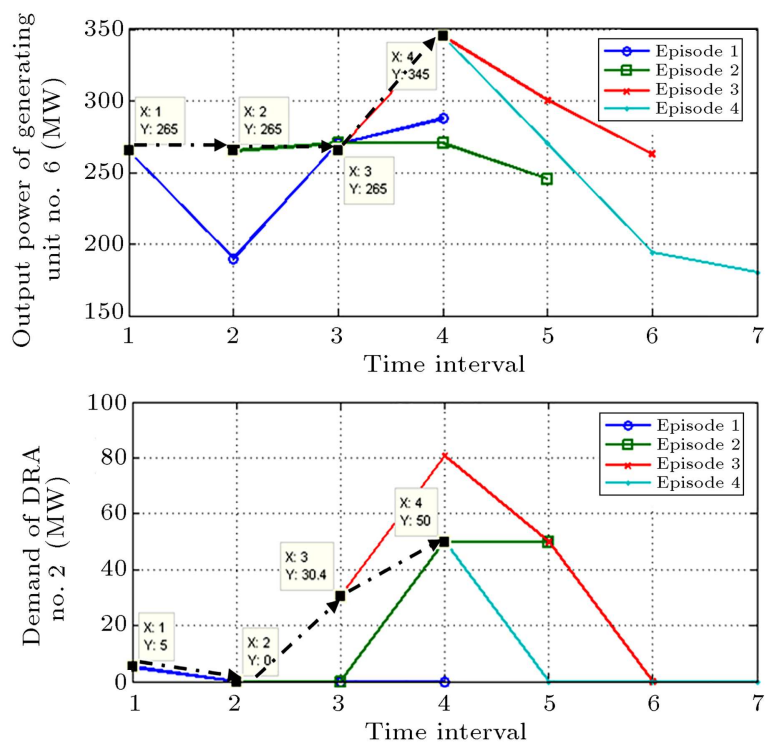

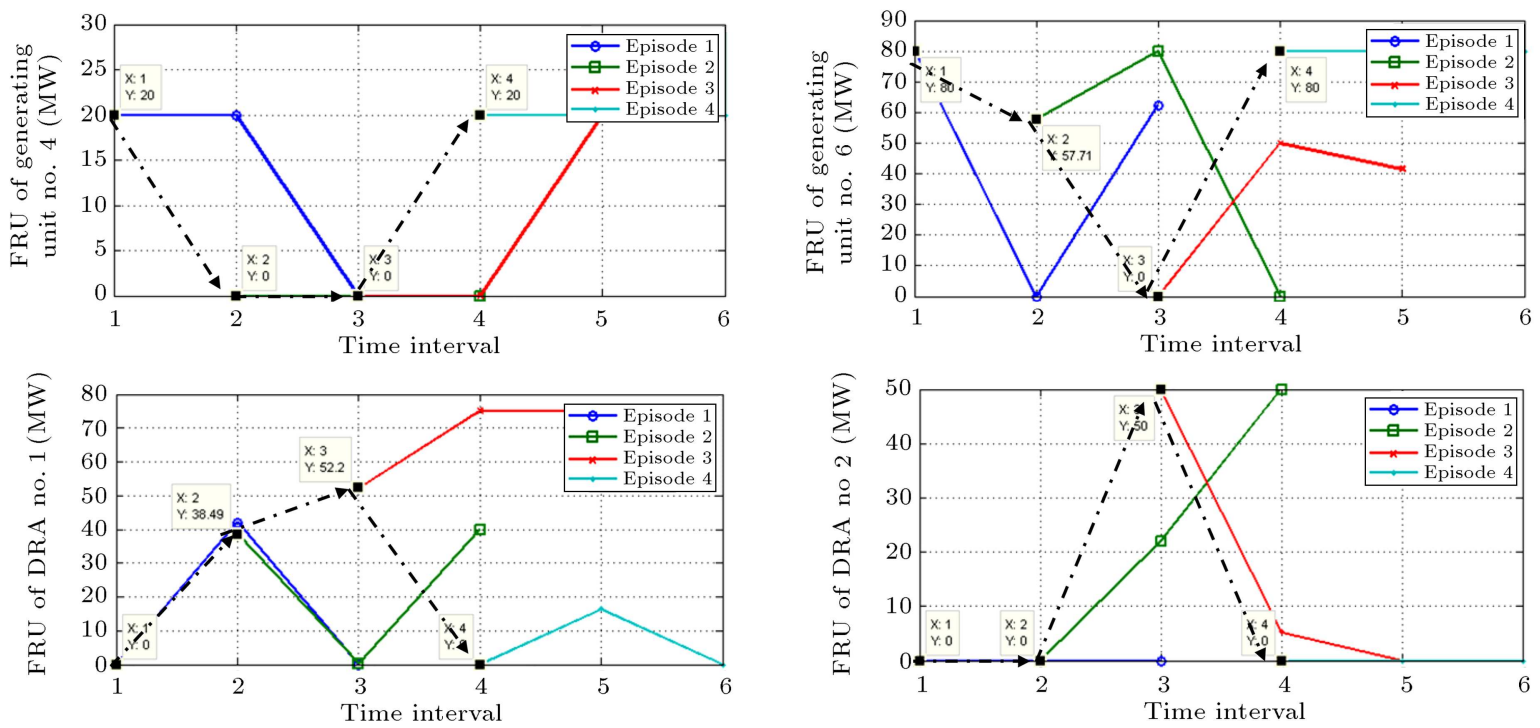

Figure 6. The FRU of the generating units 4 and 6 and DRAs. Dash-dotted arrows show the trend of the amount of power, which should be put aside for FRU in real-time operation in equilibrium.
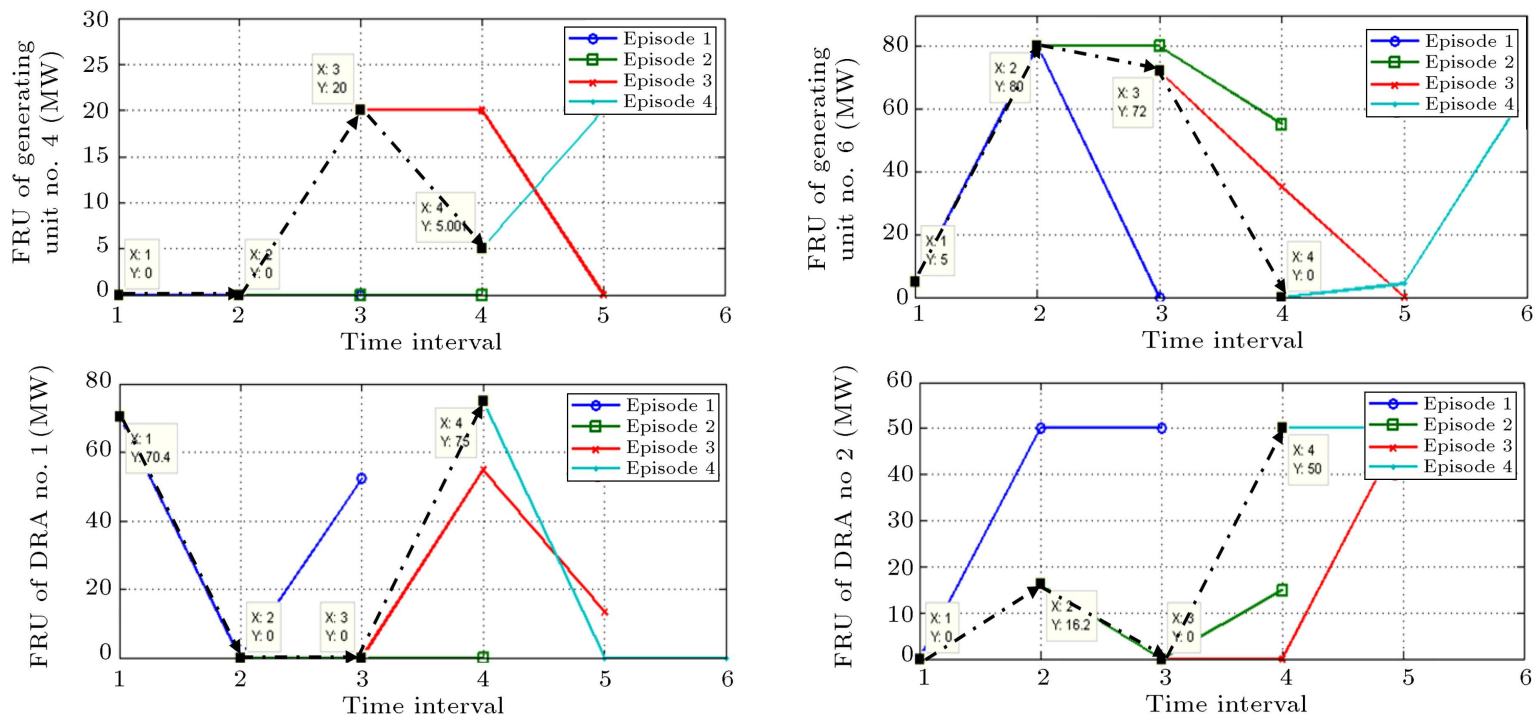

Figure 7. The FRD of the generating units 4 and 6 and DRAs. Dash-dotted arrows show the trend of the amount of power, which should be put aside for FRD in real-time operation in equilibrium.

occurs in the first and second episodes. To ensure greater flexibility, it is observed how the generating level of unit 6 possesses deeper valleys and higher peaks upon comparing it with that of the generating unit 2 .

Figure 6 shows FRU of the generating units 4 and 6 and DRAs. As seen, all the ramp capabilities of unit 6 , as the most flexible unit, are given in episodes 1 and 3 to be deployed in real-time operation. Although FRU of the first DRA is equal to its nominal ramp rate at 4 to 6 time intervals of the third episode (all other points except boundary points are called advisory points), the equilibrium level of FRU is reduced to zero at the fourth time interval in episode 4 . Given that the simulation process rolls to the next time intervals, new system conditions are revealed. Therefore, at a given time interval, which acts as the first-time interval of an episode (namely $x$ th episode), the difference between the equilibrium point, which is just achieved in the $x$ th episode, and the advisory points, which are obtained in the previous $x$ th episodes, is justified. Based on Figure 7 and regarding the FRD of the generating unit 4, the same conclusion is valid. As seen, the advisory points for the second- and third-time intervals are zero, while the associated equilibrium point takes the maximum ramping capability of the generating unit 4. Referring to FRD of DRAs in Figure 7, one can find that their maximum ramping capabilities are utilized in some time intervals. This happens because system tends to procure ramp requirement in the most economically way. 


\section{Conclusion}

A boundary condition approach to finding equilibrium was proposed in this paper where the demand response resources were actively involved to procure the flexible ramp requirement of the system. At each time interval, a security economic dispatch process was applied on a forward rolling basis in which the next three 15minute intervals were considered at each interval. The equilibrium points (boundary conditions) in each run of real-time market carried the burden of initializing the next run. In doing so, the simulation process was updated based on the new condition of the system as the real-time market goes forward. The results illustrated that demand response resources could help the system functionality in terms of ramping deficiency in the equilibrium state. Accordingly, considering penalty price for system ramp requirements might necessitate forming uplift payments.

\section{Nomenclature}

\section{Indices}

$d$

$i$

j

$t$

\section{Sets}

$\Omega_{j}$

$\Xi^{U L}$

\section{Parameters}

$\lambda_{t}^{G}$

$\lambda_{d}^{D}$

$N L_{t}^{E X}$

$\underline{P_{i}^{G}}, \overline{P_{i}^{G}}$

$\underline{P_{d}^{D}}, \overline{P_{d}^{D}}$

$R R_{i}^{G}$

$R R_{d}^{D}$

$\Delta$

FP
Index for demand response aggregators

Index for generating units

Index for firms

Index for time intervals

Set of generating units/demand response aggregators belonging to the $j$ th firm

Set of primal variables of the upper-level problem

Marginal cost of generating unit (\$/MWh)

Marginal utility of demand (\$/MWh)

Expected net-load (MW)

Generator minimum and maximum output powers (MW)

Demand minimum and maximum consumptions (MW)

Ramp rate of generating unit (MW/15min)

Ramp rate of demand (MW/15min)

Fraction of time interval duration to hour (here $15 \mathrm{~min} / 60 \mathrm{~min}$ )

Penalty price for ramp violation $(\$ / \mathrm{MWh})$
$P_{i, t 0}^{G} / P_{d, t 0}^{D}$

Initial output/ consumed power of generating unit/demand (MW)

$D R_{i, t 0}^{G}, U R_{i, t 0}^{G} \quad$ Initial flexible ramp-down and ramp-up of generating unit (MW)

$D R_{d, t 0}^{D}, U R_{d, t 0}^{D}$ Initial flexible ramp-down and ramp-up of demand (MW)

$F R D P_{t}, F R U P_{t}$ System ramp-down and ramp-up requirements in time interval $t+1$ $(\mathrm{MW} / 15 \mathrm{~min})$

\section{Variables}

$\alpha_{i, t}^{G} \quad$ Price offered by generating unit in the real-time market ( $\$ / \mathrm{MWh})$

$\alpha_{d, t}^{D} \quad$ Price offered by demand response aggregator in the real-time market $(\$ / \mathrm{MWh})$

$P_{i, t}^{G}$

$P_{d, t}^{D}$

Scheduled generating unit power in the real-time market (MW)

Scheduled demand response aggregator power in the real-time market (MW)

$D R_{i, t}^{G}, U R_{i, t}^{G} \quad$ Flexible ramp-down and ramp-up supplied by generating unit in the real-time market (MW)

$D R_{d, t}^{D}, U R_{d, t}^{D}$

Flexible ramp-down and rampup supplied by demand response aggregator in the real-time market (MW)

$\lambda_{t} \quad$ Real-time clearing market price $(\$ / \mathrm{MW} / 15 \mathrm{~min})$

$\mu_{t}^{D}, \mu_{t}^{U}$

Flexible ramp-down and ramp-up price $(\$ / \mathrm{MW} / 15 \mathrm{~min})$

$S_{t}^{D}, S_{t}^{U}$

Flexible ramp-down and ramp-up slack variables $(\mathrm{MW})$

\section{Abbreviation List}

DRA

Demand Response Aggregator

DRP

Demand Response Program

EPEC

Equilibrium Problems with Equilibrium Constraints.

FRD

Flexible Ramp-Down

FRP Flexible Ramp Product

FRU Flexible Ramp-Up

MPEC Mathematical Program with Equilibrium Constraints

NE Nash Equilibrium

RCP Ramp Capability Product 


\section{References}

1. Navid, N. and Rosenwald, G. "Ramp capability product design for MISO markets", MISO, Market Development and Analysis (2013).

2. Abdul-Rahman, K.H., Alarian, H., Rothleder, M., et al. "Enhanced system reliability using flexible ramp constraint in CAISO market", Proc. IEEE Power Energy Soc. Gen. Meeting, San Diego, USA, pp. 1-6 (2012).

3. Srinivasan, D., Trung, L.T., and Singh, C. "Bidding and cooperation strategies for electricity buyers in power markets", IEEE Syst. J., 10(2), pp. 422-433 (2016).

4. Klemperer, P.D. and Meyer, M.A. "Supply function equilibria in oligopoly under uncertainty", Econometrica: J. Econometric Soc., pp. 1243-1277 (1989).

5. Anderson, E.J. and Hu, X. "Finding supply function equilibria with asymmetric firms", Operations Research, 56(3), pp. 697-711 (2008).

6. Azadi, A. and Akbari Foroud, A. "A new solution approach for supply function equilibrium-based bidding strategy in electricity markets", Scientia Iranica, 24(6), pp. 3231-3246 (2017).

7. Rahimiyan, M. and Baringo, L. "Strategic bidding for a virtual power plant in the day-ahead and realtime markets: A price-taker robust optimization approach", IEEE Trans. Power Syst., 31(4), pp. 26762687 (2016).

8. Xu, Z., Hu, Z., Song, Y., et al. "Risk-averse optimal bidding strategy for demand-side resource aggregators in day-ahead electricity markets under uncertainty", IEEE Trans. Smart Grid, 8(1), pp. 96-105 (2017).

9. Ladjici, A.A., Tiguercha, A., and Boudour, M. "Nash equilibrium in a two-settlement electricity market using competitive co-evolutionary algorithms", Int. J. Electr. Power Energy Syst., 57, pp. 148-55 (2014).

10. Nezamabadi, N. and Vahidinasab, V. "Microgrids bidding strategy in a transactive energy market", Scientia Iranica, 26, pp. 3622-3634 (2019).

11. Bashi, M.H., Yousefi G.R., Latify, M.A., et al. "Impacts of intraday risky power trades on the high wind penetrated electricity markets", IET Gener. Transm. Distrib, 13(17), pp. 3836-3846 (2019).

12. Ruiz, C., Conejo, A.J., and Smeers, Y. "Equilibria in an oligopolistic electricity pool with stepwise offer curves", IEEE Trans. Power Syst., 27(2), pp. 752-761 (2012).

13. Cornelius, A. "Assessing the impact of flexible ramp capability products in the midcontinent ISO", Master's Thesis, Duke University (2014).

14. Bashi, MH., Gharibpour, H., Rahmati, I., et al. "Including the constraints that have less than onehour characteristics in an hourly based generating scheduling regime", IEEE International Conference on Environment and Electrical Engineering, pp. 1-4 (2018).
15. Doostizadeh, M., Aminifar, F., Ghasemi, H., et al. "Energy and reserve scheduling under wind power uncertainty: An adjustable interval approach", IEEE Trans. on Smart Grid, 7(6), pp. 2943-2952 (2016).

16. Zou, P., Chen, Q., Wang, J., et al. "Evaluating the impacts of flexible ramping products on the market equilibrium", IEEE Power Energy Soc. Gen. Meeting, San Diego, Boston, USA (2016).

17. AEMO, Integrating Renewable Energy-Wind Integration Studies Report, Melbourne, Australia, pp. 3-21 (2013).

18. Chen, R., Wang, J., Botterud, A., et al. "Wind power providing flexible ramp product", IEEE Trans. Power Syst., 32(3), pp. 2049-2061 (2017).

19. Cui, M., Zhang, J., Wu, H., et al. "Wind-friendly flexible ramping product design in multi-timescale power system operations", IEEE Trans. Sustain. Energy, 8(3), pp. 1064-1075 (2017).

20. Heydarian-Forushani, E., Golshan, M.E.H., Shafiekhah, M., et al. "Optimal operation of emerging flexible resources considering sub-hourly flexible ramp product", IEEE Trans. Sustain. Energy, 9(2), pp. 916929 (2018).

21. Fang, X., Krishnan, V., and Hodge, B.-M. "Strategic offering for wind power producers considering energy and flexible ramping products", Energies, 11(5), p. 1239 (2018).

22. Gharibpour, H. and Aminifar, F. "Multi-stage equilibrium in electricity pool with flexible ramp market", Int. J. Electr. Power Energy Syst., 109, pp. 661-671 (2019).

23. Chen, Q., Zou, P., Wu, C., et al. "A Nash-Cournot approach to assessing flexible ramping products", Appl. Energy, 206, pp. 42-50 (2017).

24. https://www.eia.gov/electricity/data/eia861/dsm/

25. York, D. and Kushler, M. "Exploring the relationship between demand response and energy efficiency: A review of experience and discussion of key issues", American Council for an Energy-Efficient Economy (2005)

26. Roozbehani, M., Dahleh, M., and Mitter, S. "Dynamic pricing and stabilization of supply and demand in modern electric power grids", IEEE Intl. Conf. Smart Grid Commun., Gaithersburg, USA (2010).

27. Tulabing, R., Yin, R., DeForest, N., et al. "Modeling study on flexible load's demand response potentials for providing ancillary services at the substation level", Electric Power Systs. Research, 140, pp. 240-252 (2016).

28. FERC, Assessment of Demand Response and Advanced Metering, Federal Energy Regulatory Commission (2006).

29. Aalami, H., Moghaddam, M.P., and Yousefi, G. "Demand response modeling considering interruptible/curtailable loads and capacity market programs", Appl. Energy, 87(1), pp. 243-250 (2010). 
30. Hu, J., Wen, F., Wang, K., et al. "Simultaneous provision of flexible ramping product and demand relief by interruptible loads considering economic incentives", Energies, 11(1), p. 46 (2017).

31. Helistö, N., Kiviluoma, J., and Holttinen, H. "Longterm impact of variable generating and demand side flexibility on thermal power generating", IET Renew. Power Gener., 12(6), pp. 718-726 (2018).

32. Hu, J., Sarker, M.R., Wang, J., et al. "Provision of flexible ramping product by battery energy storage in day-ahead energy and reserve markets", IET Gener. Transm. Distrib., 12(10), pp. 2256-2264 (2018).

33. Nikoobakht, A., Aghaei, J., Shafie-khah, M., et al. "Assessing increased flexibility of energy storage and demand response to accommodate a high penetration of renewable energy sources", IEEE Trans. Sustain. Energy, 10(2), pp. 659-669 (2019).

34. Mohammadi, J., Rahimi-Kian, A., and Ghazizadeh, M.-S. "Aggregated wind power and flexible load offering strategy", IET Renew. Power Gener., 5(6), pp. 439-447 (2011).

35. Klobasa, M. "Analysis of demand response and wind integration in Germany's electricity market", IET Renew. Power Gener., 4(1), pp. 55-63 (2010).

36. https://www.caiso.com/Documents/Section34_RealimeMarket_ asof_Feb15_2018.pdf

\section{Biographies}

Hassan Gharibpour received the BSc degree with the first class honor, 2009 from K. N. Toosi University of Technology, the MSc degree, 2011, and the PhD degree, 2020 from University of Tehran, all in Electrical Engineering. His research interests include restructuring and electricity markets, renewable energies, optimization and its application in the power systems. $\mathrm{He}$ is one of the recipients of the best reviewer award of IEEE Transactions on Sustainable Energy, 2019.

Farrokh Aminifar (Senior Member, IEEE) is currently an Associate Professor with the School of Electrical and Computer Engineering, College of Engineering, University of Tehran, Tehran, Iran, and a Senior Research Associate with the Robert W. Galvin Center for Electricity Innovation, Illinois Institute of Technology, Chicago, IL, USA. He is the recipient of the 2011 IEEE Iran Section Best PhD Dissertation Award, the 2013 IEEE/PSO Transactions Prize Paper A ward, the 2015 IEEE Iran Section Young Investigator Award, 2017 Outstanding Young Scientist Award of Iran National Academy of Science, and COMSTECH 2017 Best Young Researcher Award. Dr. Aminifar is an Editor for the IEEE TRANSACTIONS ON POWER SYSTEMS, IEEE POWER ENGINEERING LETTERS, and IET Smart Grid. His current research interests include synchrophasor applications, resilience analysis, and power system optimization. 